\title{
Gas-Expanded Liquids: Synergism of Experimental and Computational Determinations of Local Structure
}

\author{
Charles A. Eckert, Charles L. Liotta, and Rigoberto \\ Hernandez, Georgia Institute of Technology
}

\section{Final Report}

\begin{abstract}
This project focuses on the characterization of a new class of solvent systems called gasexpanded liquids (GXLs), targeted for green-chemistry processing. The collaboration has adopted a synergistic approach combining elements of molecular dynamics (MD) simulation and spectroscopic experiments to explore the local solvent behavior that could not be studied by simulation or experiment alone. The major accomplishments from this project are:
\end{abstract}

- Applied MD simulations to explore the non-uniform structure of $\mathrm{CO}_{2} /$ methanol and $\mathrm{CO}_{2} /$ acetone GXLs and studied their dynamic behavior with self-diffusion coefficients and correlation functions

- Studied local solvent structure and solvation behavior with a combination of spectroscopy and MD simulations

- Measured transport properties of heterocyclic solutes in GXLs through TaylorAris diffusion techniques and compared these findings to those of MD simulations

- Probed local polarity and specific solute-solvent interactions with Diels-Alder and $\mathrm{S}_{\mathrm{N}} 2$ reaction studies

The broader scientific impact resulting from the research activities of this contract have been recognized by two recent awards: the Presidential Green Chemistry Award (Eckert \& Liotta) and a fellowship in the American Association for the Advancement of Science (Hernandez).

In addition to the technical aspects of this contract, the investigators have been engaged in a number of programs extending the broader impacts of this project. The project has directly supported the development of two postdoctoral researcher, four graduate students, and five undergraduate students. Several of the undergraduate students were co-funded by a Georgia Tech program, the Presidential Undergraduate Research Award. The other student, an African-American female graduated from Georgia Tech in December 2005, and was co-funded through an NSF Research and Education for Undergraduates (REU) award. 


\section{Final Technical Report}

\section{Introduction}

Gas-expanded liquids (GXLs) are an environmentally-benign class of solvent systems suitable for reactions and separations. GXLs are liquid mixtures consisting of an organic solvent combined with a high-fluidity gas, such as $\mathrm{CO}_{2}$, in the nearcritical regime. GXLs offer a built-in separation mechanism where $\mathrm{CO}_{2}$ can be vented from the system leading to facile separation and recycle; therefore, they are well suited for a wide variety of applications. We have probed the cybotactic region (the region of solvent where fluid structure is influenced by solute-solvent interactions) in GXLs through a unique combination of simulations and experiments. UV/Vis and fluorescence spectroscopy experiments on pyrene and Coumarin 153 and several reactions provide indirect evidence of local heterogeneities in GXLs. Molecular dynamics (MD) simulations complement these experiments by providing both a direct picture of the microscopic structure and comparison to the experiments.

\section{Theoretical Investigations}

The solvent structure and transport properties of $\mathrm{CO}_{2}$-expanded acetone and methanol were explored with MD simulations. At low $\mathrm{CO}_{2}$ concentrations, the GXLs have similar properties as the pure organic liquids. As the $\mathrm{CO}_{2}$ concentration is increased towards the co-solvent range methanol molecules tend to cluster at distinct angles, shown with the radial distribution (Figure 1a) and the 2D spatial distribution function (Figure 2). Acetone tends to see self-interaction in a similar manner over the range of $\mathrm{CO}_{2}$ concentration as evidenced by its radial distribution function in Figure 1b. Transport properties like selfdiffusion coefficients increase with added $\mathrm{CO}_{2}$ concentration. These will be discussed later. 


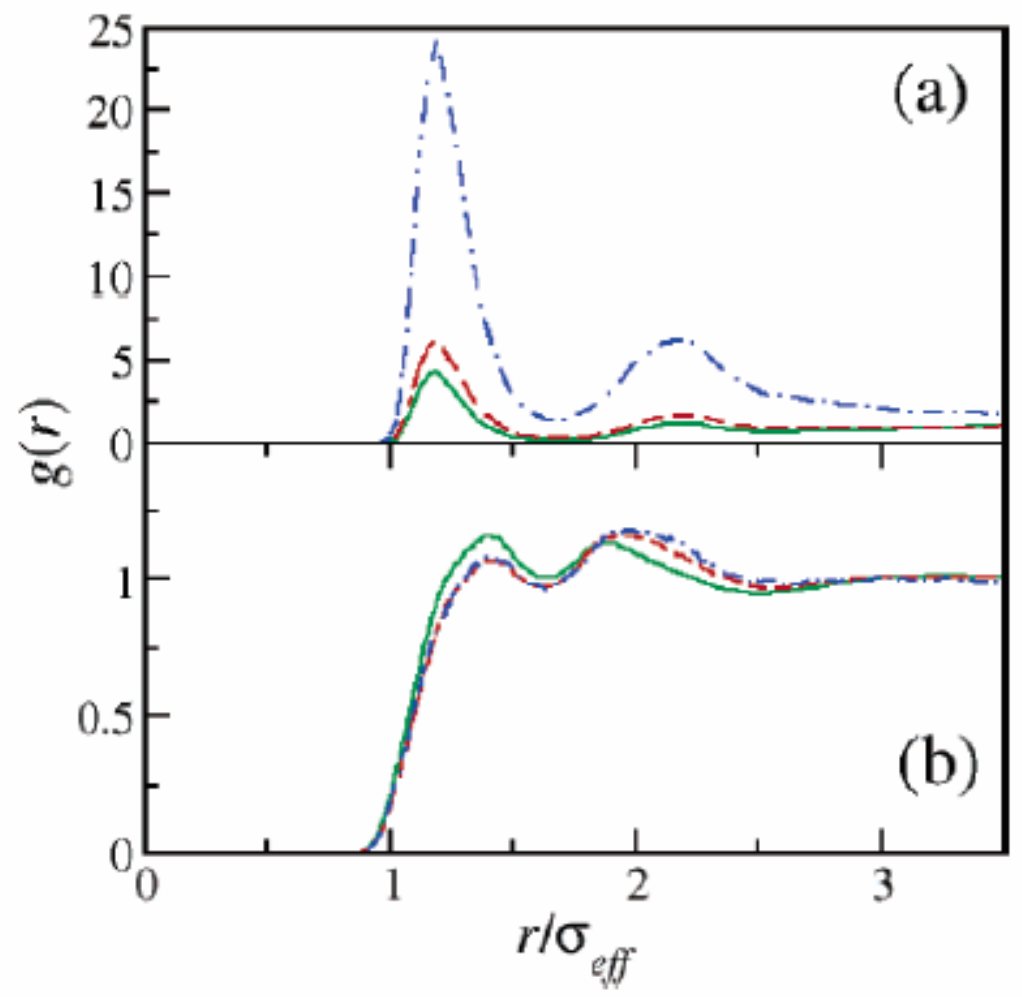

Figure 1: Radial Distribution Functions $(\mathrm{g}(\mathrm{r}))$ in $\mathrm{CO}_{2}$ expanded methanol (a) and acetone (b). Peaks correspond to most probable distances between methanol's protic hydrogen and oxygen atoms (a) and acetone's aprotic carbon and carbonyl oxygen atoms. $\sigma_{\text {eff }}$ is the effective Van der Waals interaction radius.
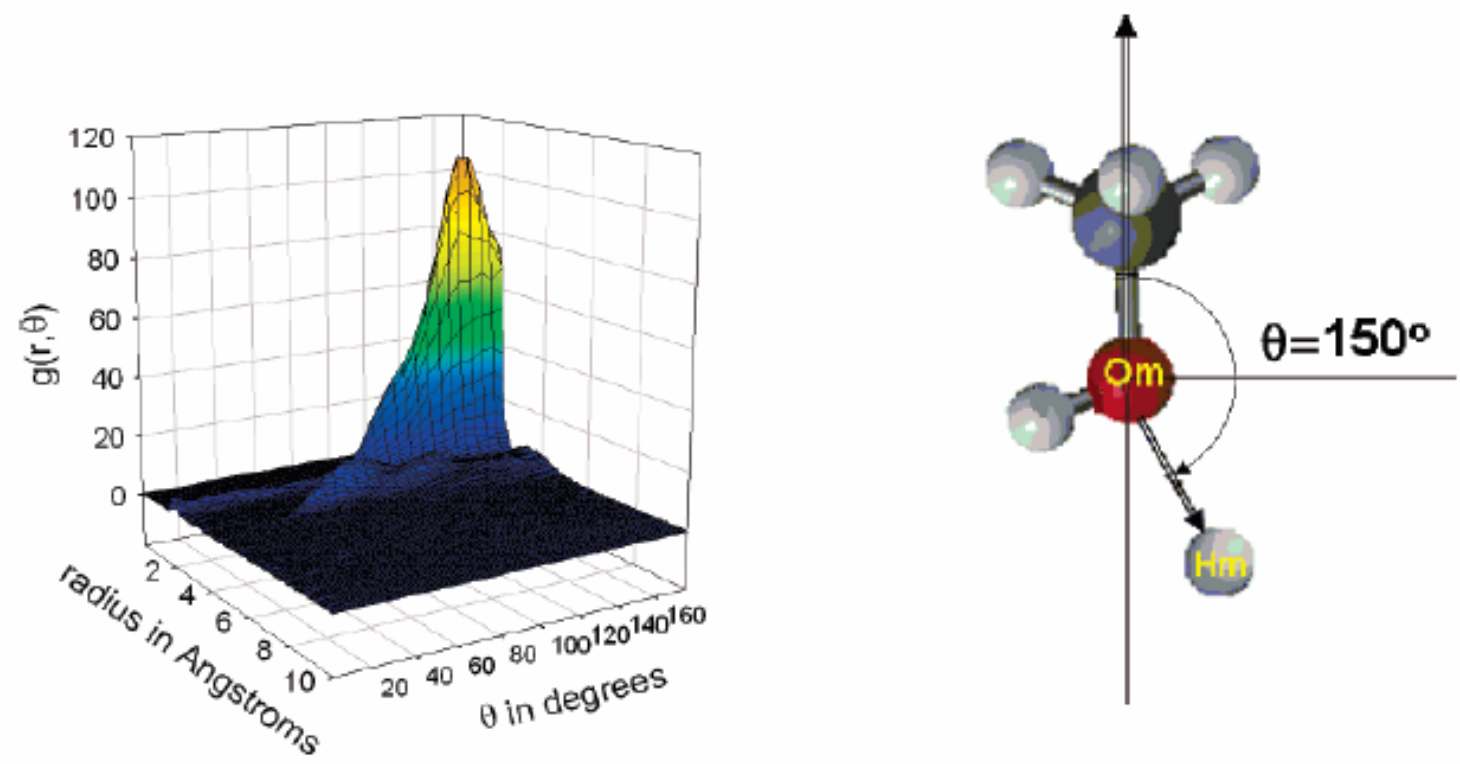

Figure 2: 2-dimensional spatial distribution function (left) of methanol shows specific orientations between the specified plane of a methanol molecule and the protic hydrogen of another methanol molecule. 
We have performed molecular dynamics (MD) simulations to determine the equilibrium solvent structure around the probe Coumarin $153(\mathrm{C} 153)$ in two GXL systems: $\mathrm{CO}_{2}$ expanded methanol and $\mathrm{CO}_{2}$-expanded acetone. These two solvent systems were chosen as an extension of our previous work on structural behavior of the same two solvents. Additionally, the hydrogen-bonding ability of methanol as well as the dipole in acetone allows us to explore the specific interactions with the $\mathrm{C} 153$ probe. Coumarin was chosen because it has optical properties that allow spectroscopic studies to compliment the theoretical calculations. Similar experimental studies were preformed on the probe pyrene in these same two GXLs as well as $\mathrm{CO}_{2}$-expanded acetonitrile.
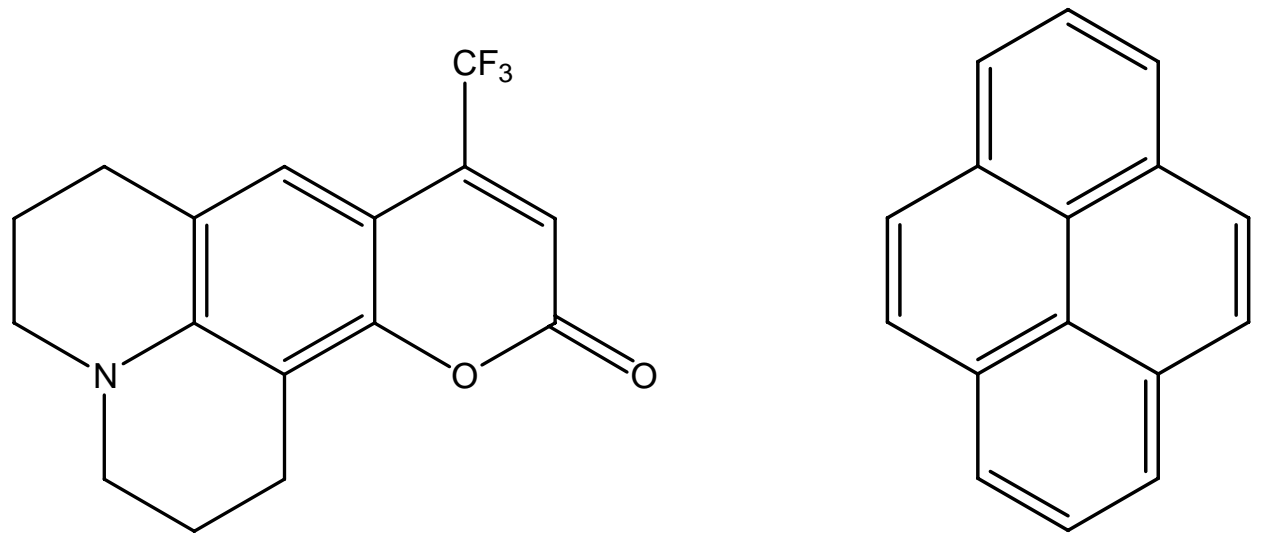

Figure 3: Structures of Coumarin 153 (left) and pyrene (right)

MD simulations were performed on both the ground and excited states ${ }^{1}$ of $\mathrm{C} 153$ in $\mathrm{CO}_{2}$ expanded methanol and acetone at three different $\mathrm{CO}_{2}$ concentrations. Figures $2 \& 3$ show the clustering behavior of methanol and acetone, respectively, around $\mathrm{C} 153$ in a plane parallel to, but approximately $3 \AA$ below, the plane of the probe molecule (represented by the large dots). The degree of organic accumulation is represented by the background color. The color gradations - ranging from blue to red - correspond to highly probable regions of bulk organic concentration (blue) and local organic concentration (red). Two salient points are inferred from Figures 3 and 4: a) the cybotactic region around $\mathrm{C} 153$ in $\mathrm{CO}_{2}$-expanded methanol shows enhanced concentrations of methanol near the acceptor side of the molecule (the side with oxygen and fluorine atoms) b) methanol molecules cluster equivalently above and below the plane particularly on the acceptor side. The preferential clustering near the acceptor side is consistent with strong specific and dipole-dipole, solute-solvent interactions. 


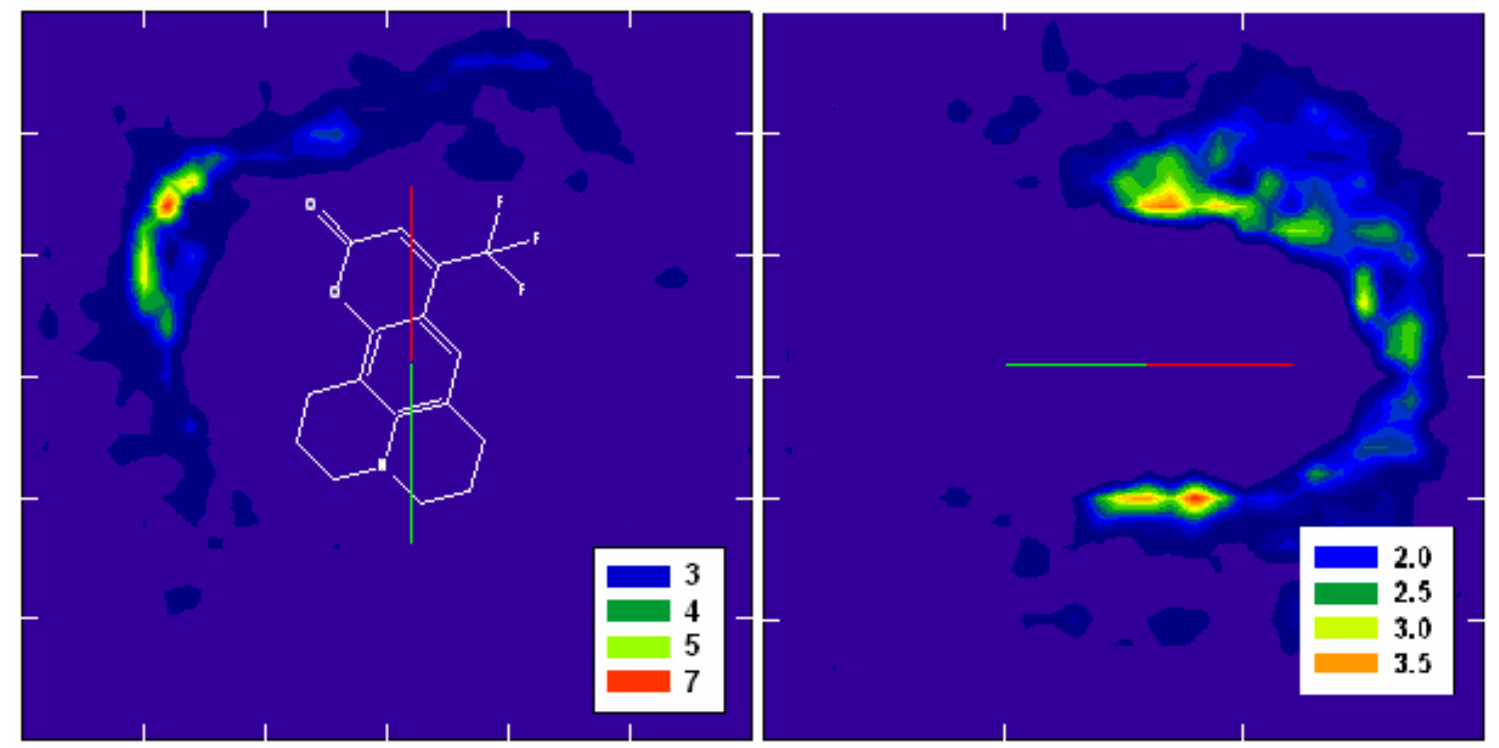

Figure 3 and 4: Accumulation of methanol around Coumarin 153 in $\mathrm{CO}_{2}$-expanded methanol from two different vantage points: a bird's eye view into the Coumarin 153 Plane (left) and above and below the Coumarin probe (right). Degree of solvent clustering relative to the bulk composition ( $5 \%$ methanol is provided by the scale). 


\section{Diffusion Coefficients}

In addition to local structural information, diffusion coefficients in $\mathrm{CO}_{2}$-expanded methanol and $\mathrm{CO}_{2}$-expanded acetone at $298 \mathrm{~K}$ were calculated using molecular dynamics simulations. Figure 5 displays simulated diffusion coefficients of i) methanol in $\mathrm{CO}_{2}$ expanded methanol and ii) acetone in $\mathrm{CO}_{2}$-expanded acetone. The simulated results correctly predict an increase in diffusion with $\mathrm{CO}_{2}$ addition. Experimental NMR points ${ }^{2}$ for pure methanol and acetone are plotted in Figure 5 for reference. The simulated diffusion coefficient for pure methanol at $298 \mathrm{~K}$ is somewhat smaller than the experimental value, ${ }^{\text {and }}$ the simulated pure acetone diffusion is a bit greater. Although our values for diffusion coefficients are not in exact agreement with experimental values, the models reproduce the general trend of enhanced solvent diffusivity with $\mathrm{CO}_{2}$ addition.

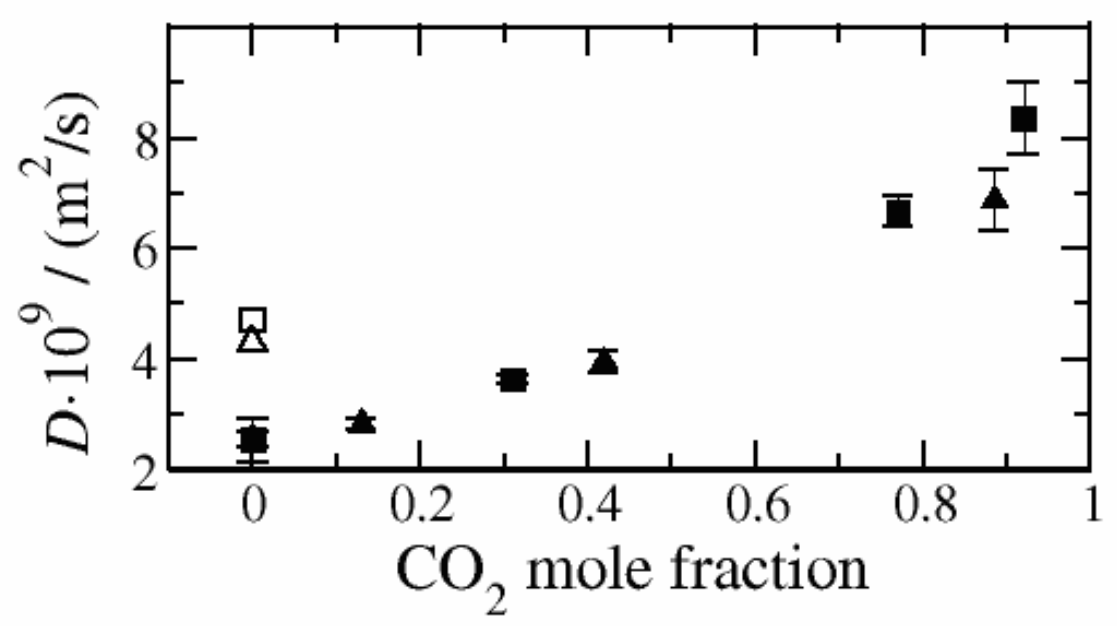

Figure 5: Simulated self-diffusion coefficients of $(\boldsymbol{\Delta})$ methanol in $\mathrm{CO}_{2}$-expanded methanol and ( $\mathbf{\square})$ Acetone in $\mathrm{CO}_{2}$-expanded acetone at $298 \mathrm{~K}$. Self-diffusion coefficients determined experimentally by $\mathrm{NMR}^{2}$ for $(\square)$ pure acetone and $(\Delta)$ pure methanol are included. Error bars in the diffusion coefficients were determined by root-mean-square deviations from the average value.

Figure 6 presents both simulated and experimental diffusion coefficients of pyridine, pyrimidine, pyrazine, and benzene solutes versus volume fraction of $\mathrm{CO}_{2}$ in $\mathrm{CO}_{2}$-expanded methanol. Experimental diffusion coefficients were determined using the Taylor-Aris dispersion technique ${ }^{3-5}$. Each solute group contains simulated and experimental diffusion coefficients at three volume fraction $\mathrm{CO}_{2}: 0 \%, 25 \%$, and $75 \%$. The simulated diffusion coefficients of all four solutes increase with added $\mathrm{CO}_{2}$ pressure, a finding consistent with the experimental results. The average simulated diffusion coefficients shown in these tables agree reasonably well with the experimental value. Solvatochromic studies are being conducted to investigate the local solvent structure around the probes to determine if a specific interaction may affect diffusion. 


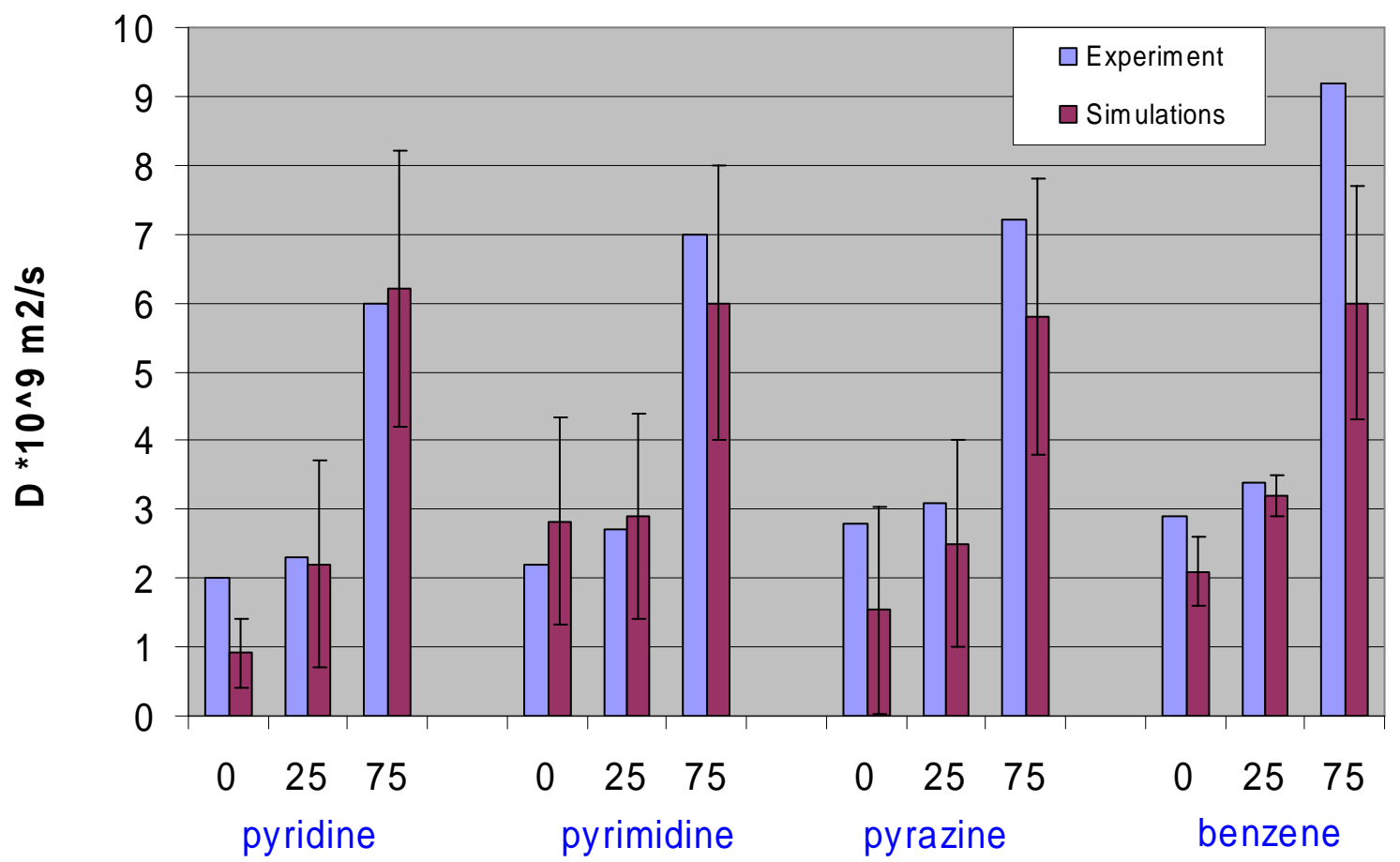

\section{volume \% carbon dioxide}

Figure 6: Diffusion coefficients of benzene, pyridine, pyrimidine, pyrazine in $\mathrm{CO}_{2}$ expanded methanol for $0 \%, 25 \%$ and $75 \%$ volume fraction carbon dioxide from TaylorAris dispersion experiments and simulations. $\mathrm{T}=313 \mathrm{~K}, \mathrm{P}=150$ bar. Error bars on the simulations were determined from root mean squared deviations from the average value. 


\section{Experimental Work}

\section{Solvatochromic Investigations}

$\mathrm{UV} / \mathrm{Vis}$ and fluorescence solvatochromic studies were performed on C153 in $\mathrm{CO}_{2}$-expanded methanol and acetone as an experimental counterpart to the theoretical investigations. The wavelength at maximum absorption and emission was monitored as a function of $\mathrm{CO}_{2}$ concentration in the two GXLs used in the theoretical investigations. The sensitivity of the maximum wavelengths to local composition is shown in Figure 7. As $\mathrm{CO}_{2}$ is added to the organic solvent, we see a gradual decrease in wavelength maximum until very high $\mathrm{CO}_{2}$ concentration, where there occurs a steep decrease as the concentration approaches pure $\mathrm{CO}_{2}$. We are now using these optical results to estimate local compositions of the solvent mixture around the probe.

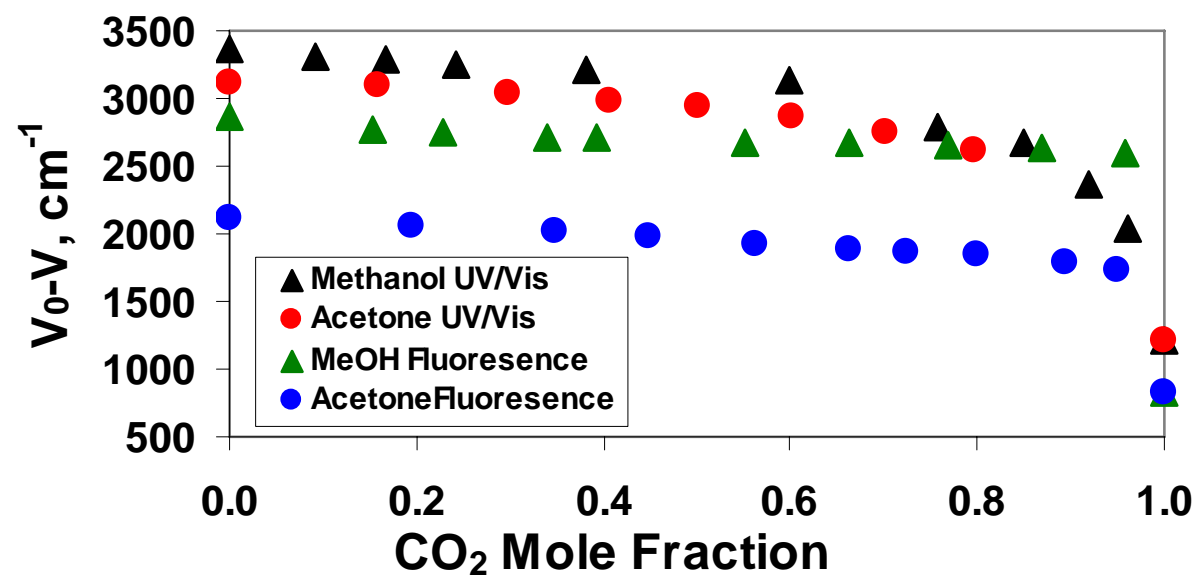

Figure 7: UV/Vis and fluorescence spectroscopy results for Coumarin 153 in $\mathrm{CO}_{2}$ expanded methanol and acetone at $300 \mathrm{~K}$.

Pyrene fluorescence was used as a tool to probe local polarity. Pyrene has a total of five characteristic vibronic bands in its emission spectrum (Figure 8a). The intensity of the first band is strongly dependent on solvent polarity, while that of the third band is insensitive to the surroundings. The ratio of the first to the third band $\left(\mathrm{Py}=\mathrm{I}_{1} / \mathrm{I}_{3}\right)$ defines the Py scale, plotted in Figure $8 \mathrm{~b}$ vs. mole fraction of $\mathrm{CO}_{2}$ for three different GXL systems. We notice a precipitous decline in Py for both acetone and acetonitrile GXLs at 0.6 mole fraction, with a more subtle decline in the methanol GXL. The data indicate a decrease in local polarity with addition of $\mathrm{CO}_{2}$ and suggest a reordering or restructuring of the cybotactic region.
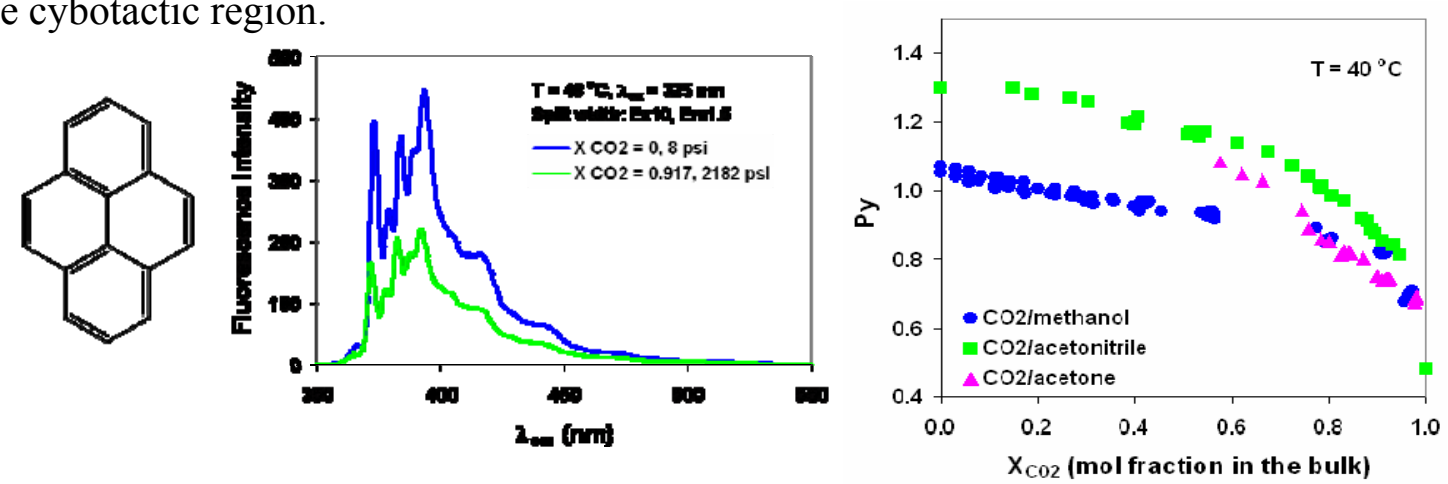
Figure 8: (a) Characteristic Vibronic Bands of Pyrene (b) Py vs. Mole Fraction $\mathrm{CO}_{2}$ in $\mathrm{CO}_{2}$-expanded methanol, acetonitrile, and acetone systems.

\section{Kinetic Studies}

We studied the effects of local interactions on the reaction rate of the Diels Alder addition of anthracene to PTAD (4-phenyl-1,2,4-triazoline-3,5-dione) in $\mathrm{CO}_{2}$-expanded acetonitrile (Figure9). The pseudo-first order rate constant is sensitive to changes in local polarity, and we explored the rate as a function of $\mathrm{CO}_{2}$ concentration. The rate constants are plotted as a function of $\mathrm{CO}_{2}$ concentration in Figure 10. The results show that the reaction rate increases modestly with $\mathrm{CO}_{2}$ concentration with a steep increase as we approach the pure $\mathrm{CO}_{2}$ limit. The rate enhancement with $\mathrm{CO}_{2}$ concentration was unexpected, because a decrease in solvent polarity (from $\mathrm{CO}_{2}$ addition) would usually cause a decrease in rate. The rate increase is attributed to a $\mathrm{CO}_{2} /$ acetonitrile complex that causes the oxygen atoms on $\mathrm{CO}_{2}$ to become more electronegative and increases the Lewis acidity and basicity of $\mathrm{CO}_{2}$. Strong Lewis acid/base interactions between $\mathrm{CO}_{2}$ and PTAD's carbonyl groups lower the transition state energy and thus increases reaction rate. Literature supports this argument ${ }^{6}$.<smiles>c1ccc2cc3ccccc3cc2c1</smiles>

Anthracene

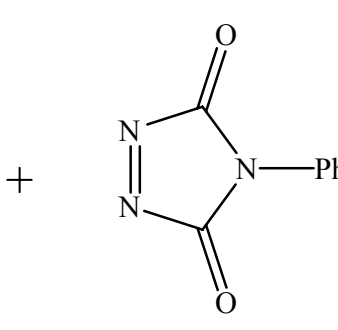

PTAD

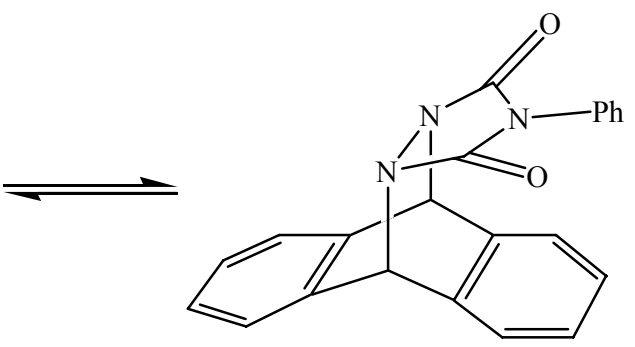

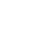




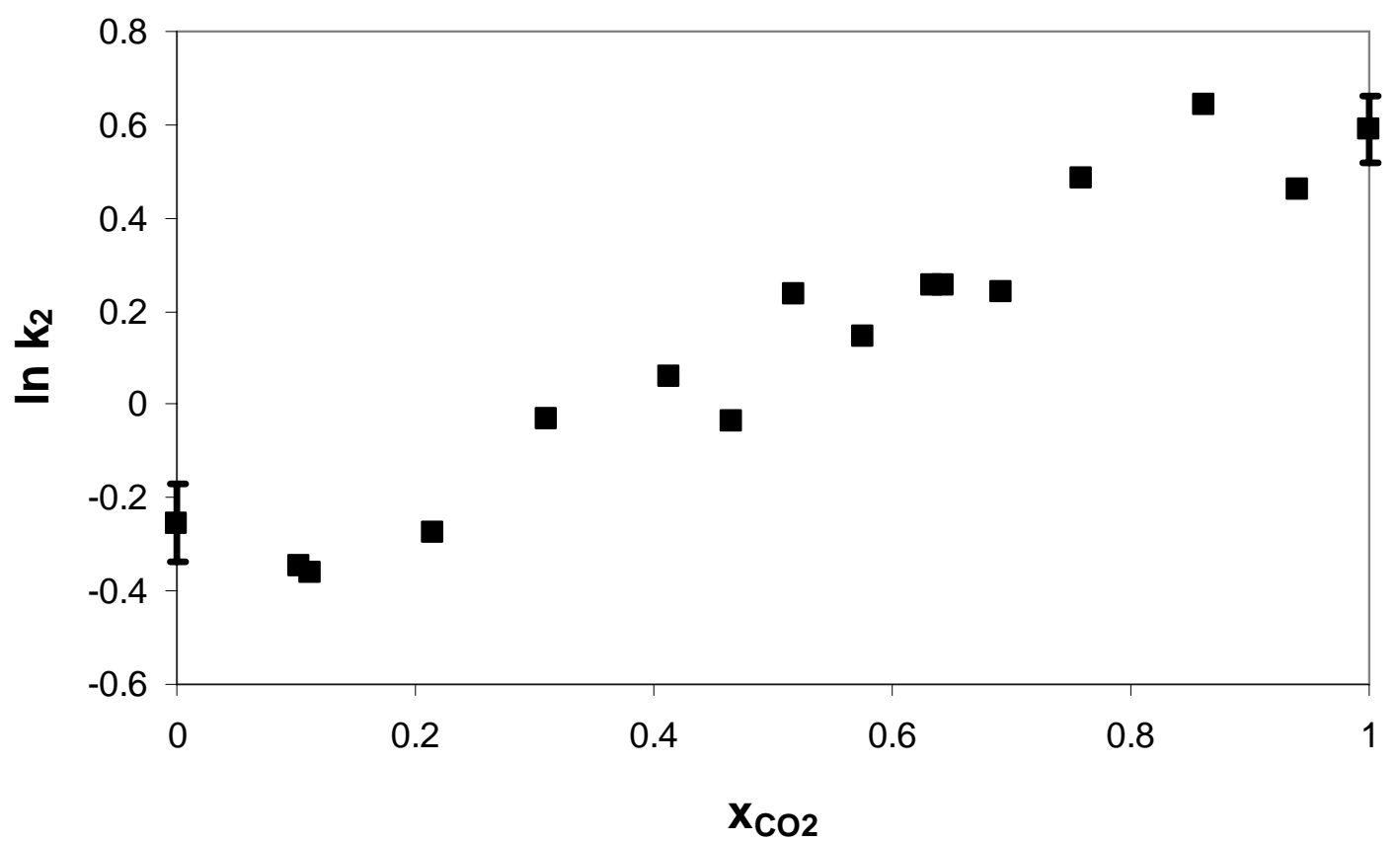

Figure 10: Pseudo first order rate constant in $\mathrm{CO}_{2}$-expanded acetonitrile for Diels-Alder reaction of anthracene and PTAD.

The reaction of tributylamine (TBA) with methyl p-nitrobenzenesulfonate (MNBS) is an $\mathrm{S}_{\mathrm{N}} 2$ nucleophilic substitution reaction (See Figure 11) that is affected by the solvent. The highly polar transition state (Figure 12) is stabilized by the polarity of solvent molecules in the immediate vicinity. Consequently, this reaction serves as an excellent probe of the local solvent polarity.

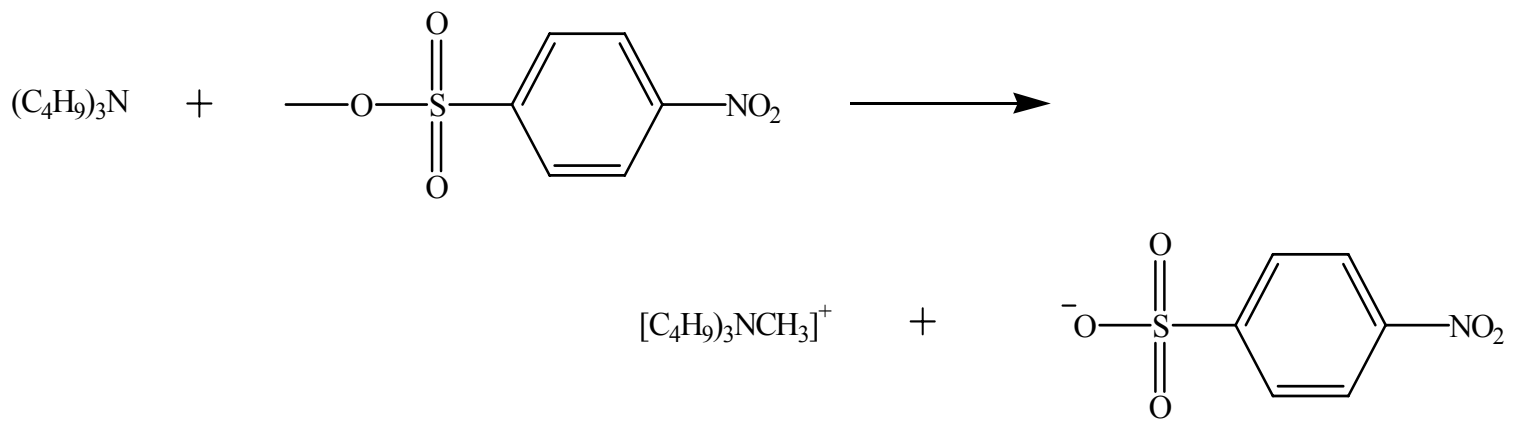

Figure 11: $\mathrm{S}_{\mathrm{N}} 2$ reaction of TBA with MNBS.<smiles>O=[N+]([O-])c1ccc(S(=O)(=O)O[C@H]([O-])[C@@H](Br)[N+](Br)(Br)Br)cc1</smiles> 
Figure 12: Polar transition state of the reaction of TBA with MNBS

Kinetic results are shown in Figure 13. As expected, the second order rate constant of the reaction decreases at increasing $\mathrm{CO}_{2}$ composition at both $25^{\circ} \mathrm{C}$ and $40^{\circ} \mathrm{C}$. The addition of $\mathrm{CO}_{2}$ reduces the polarity of the solvent, slowing down the reaction. Initial results at $25^{\circ} \mathrm{C}$ also indicate a slight increase in reaction rate at very low $\mathrm{CO}_{2}$ compositions, followed by a sharp decrease at higher $\mathrm{CO}_{2}$ compositions. This maximum was attributed to either experimental uncertainty, density enhancements in the GXL, or the large activation volume of the transition state of the reaction. 


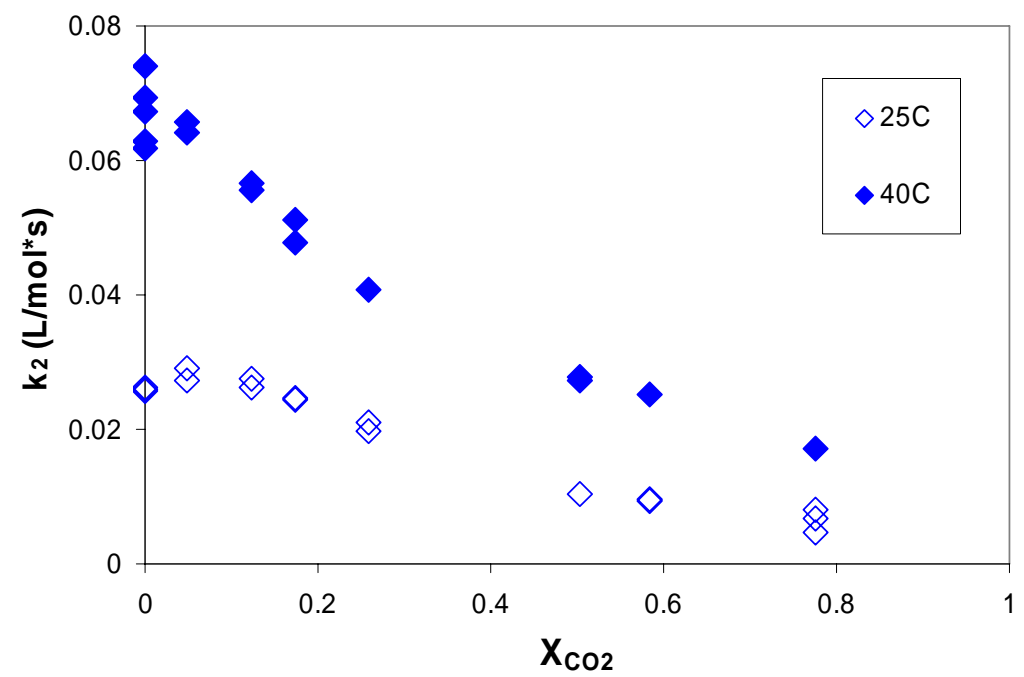

Figure 13: Second-order rate constants as a function of composition for the $\mathrm{S}_{\mathrm{N}} 2$ reaction of TBA and MNBS in gas-expanded acetonitrile at $25^{\circ} \mathrm{C}$ and $40^{\circ} \mathrm{C}$

\section{Impact of the Work}

A variety of different computational and experimental studies have been performed to better understand GXLs on a molecular level. GXLs are a potential "green" alternative to many traditional organic solvents with added benefits such as a built-in separation mechanism and pressure-tunable solvent properties. These properties make GXLs a versatile solvent for a variety of applications ranging from traditional applications as reactions and extractions to more specialized uses including gas-antisolvent crystallization and nanomaterials processing. The research activities supported by this grant have answered fundamental questions regarding solvent-solvent and solvent-solute interactions and their effects on macroscopic observations such as phase behavior, reaction kinetics, and absorption/emission spectroscopy. These important findings will enable future researchers to design and select optimal GXLs and process conditions that best suit a particular application, especially as the need for cleaner processing technologies emerges. 


\section{Relevant Publications}

There are several recent or pending publications related to this grant:

- Jie Lu, Michael J. Lazzaroni, Jason P. Hallett, Andreas S. Bommarius, Charles L. Liotta, Charles A. Eckert, "Tunable Solvents for Homogeneous Catalyst Recycle," I\&EC Res., 43 1586-1590, (2004).

- Xiaofeng Xie, Charles L. Liotta, Charles A. Eckert, " $\mathrm{CO}_{2}$ Catalyzed Acetal Formation in $\mathrm{CO}_{2}$-Expanded Methanol and Ethylene Glycol," I\&EC Res., 43, $2605-2609,(2004)$.

- Xiaofeng Xie, Charles L. Liotta, Charles A. Eckert, " $\mathrm{CO}_{2}$ Protected Amine Formation from Nitrile and Imine Hydrogenation in Gas-Expanded Liquids," I\&EC Res. 43, 7907, (2005).

- Charles A. Eckert, Charles L. Liotta, David Bush, James Brown, Jason Hallett, "Sustainable Reactions in Tunable Solvents," J Phys Chem B, 108, 18108-18118, 2004.

- E. Hershkovitz and R. Hernandez; "Chemical reaction dynamics within anisotropic solvents in time-dependent fields," J. Chem. Phys., 122, 014509, 2005

- Michael J. Lazzaroni, David Bush, James S. Brown, and Charles A. Eckert, "High Pressure Vapor + Liquid Equilibria of Some Carbon Dioxide + Organic Binary Systems," J Chem Eng Data, 50, 60, (2005).

- Michael J. Lazzaroni, David Bush, Rebecca Jones, Jason P. Hallett, Charles L. Liotta, and Charles A. Eckert, "High Pressure Phase Equilibria of Some Carbon Dioxide- Organic-Water Systems," Fluid Phase Equil, 224, 143-154, 2004.

- Jason P. Hallett, James S. Brown, Pamela Pollet, Jie Lu, Colin A. Thomas, Rebecca S. Jones, Charles L. Liotta, Charles A. Eckert, "Tunable Fluids for Sustainable Recycle of Homogeneous Catalysts," Proceedings of the $7^{\text {th }}$ Italian Conference on Supercritical Fluids and Their Applications, 2004.

- Victor T. Wyatt, David Bush, Jie Lu, Jason P. Hallett, Charles L. Liotta, Charles A. Eckert, "Determination of Solvatochromic Solubility Parameters for the Characterization of Gas-Expanded Liquids," J. Supercritical Fl, 36, 16-22, 2005.

- M. Vogt and R. Hernandez; "An idealized model for nonequilibrium dynamics in molecular systems," J.Chem. Phys., 123, 144109, (2005).

- T. Bartsch, T.Uzer and R. Hernandez, "Stochastic transition states: Reaction geometry amidst noise," J.Chem. Phys., 123, 204102, (2005).

- T. Bartsch, T. Uzer, J. M. Moix and R. Hernandez; "Identifying reactive trajectories using a moving transition state," J. Chem. Phys. 124, 244310 (2006).

- Weikel, R.R., Hallett, J.P., Liotta, C.L., and Eckert, C.A. , "Self-neutralizing in situ acid catalysts from CO2," Topics in Catalysis, 37 (2-4), 75-80 (2006)

- Shukla, C. L., Hallett, J. P., Popov, A.V., Liotta, C.L., Eckert, C.A. "Molecular Dynamics Simulation of the Cybotactic Region in Gas-Expanded MethanolCarbon Dioxide and Acetone-Carbon Dioxide Mixtures," J. Phys. Chem. B., 110, 24101-24111 (2006). 
- Hallett, J.P., Kitchens, C.L., Hernandez, R., Liotta, C.L., Eckert, C.A. "Probing the Cybotactic Region in Gas-Expanded Liquids (GXLs)," Acc. Chem. Res., 39, 531 (2006).

- Jackson W. Ford, Jie Lu, Charles L. Liotta, Charles A. Eckert. "Solvent effects on the kinetics of a Diels-Alder reaction in gas-expanded liquids." In Press, Ind. Eng. Chem. Res.

\section{References}

1. $\quad$ Kumar, P., Maroncelli, M. J. Chem. Phys. 1995, 103, 3038.

2. $\quad$ McCall, D.; Douglass, D.; Anderson, E. J. Chem. Phys. 1959, 31, 1555.

3. Taylor, G., Proceedings of the Royal Society of London. Series A, Mathematical and Physical Sciences 1953, 219, 186.

4. Aris, R., Proceedings of the Royal Society of London. Series A, Mathematical and Physical Sciences 1954, 235, 67.

5. Aris, R., Proceedings of the Royal Society of London. Series A, Mathematical and Physical Sciences 1956, 235, 67.

6. Williams, H. L.; Rice, B. M.; Chabalowski, C. F., Investigation of the $\mathrm{CH}_{3} \mathrm{CN}$ $\mathrm{CO}_{2}$ potential energy surface using symmetry-adapted perturbation theory. $J$. Phys. Chem. A 1998, 102, 6981. 